ERRATUM

\title{
Carbon-based metal-free catalysts
}

Xien Liu and Liming Dai

Nature Reviews Materials 1, 16064 (2016)

In the originally published version of this article, the corresponding authorship was incorrectly assigned. This has been corrected in both the HTML and PDF versions of the article. 


\section{ONLINE ONLY}

\section{Subject terms}

Physical sciences / Materials science / Materials for energy and catalysis / Electrocatalysis

[URI /639/301/299/886]

Physical sciences / Chemistry / Catalysis / Electrocatalysis

[URI /639/638/77/886]

Physical sciences / Chemistry / Electrochemistry / Electrocatalysis

[URI /639/638/161/886]

Physical sciences / Materials science / Materials for energy and catalysis / Electrochemistry / Electrocatalysis

[URI /639/301/299/161/886]

Physical sciences / Materials science / Nanoscale materials / Structural properties

[URI /639/301/357/537] 